\title{
A DUAL FINITE ELEMENT APPROACH FOR STRESSES OF ELASTO-PERFECTLY PLASTIC BODIES
}

\author{
P. NEITTAANMÄKI, V. RIVKIND, AND G. SEREGIN
}

\begin{abstract}
Primal and dual approaches are introduced for the elasto-perfectly plastic problems. We prove theorems for approximating the stresses of elasticperfectly plastic bodies.
\end{abstract}

\section{INTRODUCTION}

The stress state of a perfect elastic-plastic body is determined by solving a problem which is known as the Haar-Karman variational principle (see, for instance, [3]). According to the modern theory of duality the corresponding problem may be interpreted as the dual problem to the variational problem for displacement fields (the direct problem) (see [3]). Unfortunately, its functional has linear growth at infinity relative to the deviator of strain tensor. For this reason solutions with jumps of discontinuities can appear (see [9]). Possible nonregularity of solutions in the direct problem makes it difficult to obtain a priori error estimates for numerical schemes (finite element method, for example). However, the solution of the dual variational problem is known to be smoother. In particular, the solution belongs to the Sobolev space $W_{\mathrm{loc}}^{1,2}$. From the mechanical point of view, solving a dual variational problem is more preferable since elastic and plastic zones are determined by the yield condition expressed in terms of the stress tensor.

In this work we consider primal and dual approaches for elasto-perfectly plastic problems. We prove that the approximate solution of this problem converges to the exact solution, and we give an error estimate.

Finally, it should be noted that ideas used in the present paper may be applied also to other variational problems whose functionals have linear growth (such as the minimal surface problem, the Plateau problem, etc.).

\section{Primal AND DUAL FORMUlations}

Consider an elastic-plastic body being in equilibrium under action of the given forces and occupying a domain $\Omega$ in $\mathbb{R}^{n}(n=2$ or $n=3)$. We suppose that the boundary of $\Omega$ is Lipschitz continuous. The direct variational problem reads as follows.

Received by the editor November 5, 1992 and, in revised form, January 24, 1994.

1991 Mathematics Subject Classification. Primary 65N15, 73E05, 73V25, 35A15, 35J60.

Key words and phrases. Primal, dual approach, FEM, error estimate, elasto-perfectly plastic bodies.

(c) 1995 American Mathematical Society 
Problem $(\mathscr{P})$ : Find a displacement field $u \in V_{0}+u_{0}$ such that

$$
I(u)=\inf \left\{I(v): v \in V_{0}+u_{0}\right\} .
$$

Here,

$$
I(v):=\int_{\Omega} g(\varepsilon(v)) d x-M(v)
$$

and the integrand of the problem has the form

$$
g(\varepsilon):=\frac{1}{2} K_{0} \operatorname{tr}^{2} \varepsilon+g_{0}\left(\left|\varepsilon^{D}(v)\right|\right) \quad \forall \varepsilon \in \mathbb{M}_{s}^{n \times n}
$$

with

$$
g_{0}(t)= \begin{cases}\mu t^{2} & \text { if }|t| \leq t_{0}:=\frac{\sqrt{2} k_{*}}{2 \mu}, \\ \mu\left(2|t| t_{0}-t_{0}^{2}\right) & \text { if }|t| \geq t_{0} .\end{cases}
$$

Above, $K_{0}, \mu, k_{*}$ are positive constants, $\varepsilon^{D}:=\varepsilon-\frac{1}{n} \operatorname{tr} \varepsilon \mathbb{I}$ denotes the deviator of the matrix $\varepsilon \in \mathbb{M}_{s}^{n \times n}$ (the space of all symmetric $(n \times n)$ matrices), $\operatorname{tr} \varepsilon$ means the trace of $\varepsilon$ and $\mathbb{I}$ is the identity matrix in $\mathbb{M}_{s}^{n \times n}$. Moreover,

$$
M(v):=\int_{\Omega} f \cdot v d x+\int_{\partial_{2} \Omega} F \cdot v d l, \quad v \in W^{1,2}\left(\Omega ; \mathbb{R}^{n}\right),
$$

where $f, F$ are the given volume and surface loads, respectively, $u_{0}$ is a given function,

$$
V_{0}:=\left\{v \in W^{1,2}\left(\Omega ; \mathbb{R}^{n}\right): v=0 \text { in } \partial_{1} \Omega\right\},
$$

and $\partial_{1} \Omega, \partial_{2} \Omega$ are parts of the boundary $\partial \Omega$ so that

$$
\partial_{1} \Omega \cap \partial_{2} \Omega=\varnothing, \quad \overline{\partial_{1} \Omega} \cap \overline{\partial_{2} \Omega}=\Omega .
$$

We assume that

$$
u_{0} \in W^{1,2}\left(\Omega ; \mathbb{R}^{n}\right), \quad f \in L^{n}\left(\Omega ; \mathbb{R}^{n}\right), \quad F \in L^{\infty}\left(\Omega ; \partial_{2} \Omega\right),
$$

where $W^{p, q}$ and $L^{q}$ are usual Sobolev and Lebesgue spaces, respectively.

Together with the Problem $(\mathscr{P})$ we shall consider the variational problem for determining displacement fields in plasticity theory with small linear hardening $\delta \in(0, \mu]$.

Problem $\left(\mathscr{P}_{\delta}\right)$ : Find $u^{\delta} \in u_{0}+V_{0}$ such that

$$
I_{\delta}\left(u^{\delta}\right)=\inf \left\{I_{\delta}(v): v \in V_{0}+u_{0}\right\} .
$$

Here,

$$
I_{\delta}(v):=\int_{\Omega} g_{\delta}(\varepsilon(v)) d x-M(v)
$$

and

$$
g_{\delta}(\varepsilon):=\frac{1}{2} K_{0} \operatorname{tr}^{2} \varepsilon+g_{0 \delta}\left(\left|\varepsilon^{D}(v)\right|\right), \quad g_{0 \delta}(t):=\frac{1}{2} \delta t^{2}+\frac{2 \mu-\delta}{2 \mu} g_{0}(t)
$$

for $\varepsilon \in \mathbb{M}_{s}^{n \times n}, t \in \mathbb{R}$. It is well known that under conditions (2.2) Problem $\left(\mathscr{P}_{\delta}\right)$ possesses a unique solution $u^{\delta}$ (see, for example, $[3,13]$ ).

Now we can formulate problems which are dual for problems $(\mathscr{P})$ and $\left(\mathscr{P}_{\delta}\right)$, respectively. 
Problem $\left(\mathscr{P}^{*}\right)$ : Find a stress tensor $\sigma \in Q_{f} \cap K$ such that

$$
R(\sigma):=\sup \left\{R(\tau): \tau \in Q_{f} \cap K\right\}
$$

Here,

$$
R(\tau):=\int_{\Omega}\left(\varepsilon\left(u_{0}\right): \tau-\frac{1}{2} a(\tau, \tau)\right) d x-M\left(u_{0}\right)
$$

where

$$
a(\tau, \sigma):=\frac{1}{n^{2} K_{0}} \operatorname{tr} \tau \operatorname{tr} \sigma+\frac{1}{2 \mu} \tau^{D}: \sigma^{D}
$$

with $\tau$ and $\sigma$ from $\mathbb{M}_{s}^{n \times n}$. Moreover,

$$
K:=\left\{\tau \in L^{2}\left(\Omega ; M_{s}^{n \times n}\right): \mathscr{F}(\sigma(x)) \leq 0 \text { for a.a. } x \in \Omega\right\}
$$

denotes the set of admissible stress tensors satisfying the Mises yield condition and

$$
Q_{f}:=\left\{\tau \in L^{2}\left(\Omega ; M_{s}^{n \times n}\right): \int_{\Omega} \tau: \varepsilon(v) d x=M(v) \quad \forall v \in V_{0}\right\}
$$

denotes the set of all stress tensors satisfying equilibrium equations in stresses.

Remark. In this case, $\mathscr{F}: \mathbb{M}^{n \times n} \rightarrow \mathbb{R}$ is defined as follows:

$$
\mathscr{F}(\tau):=\left|\tau^{D}\right|-\sqrt{2} k_{*} \text { for } \tau \in \mathbb{M}_{s}^{n \times n} \text {. }
$$

If we suppose that the condition

$$
\exists \sigma^{1} \in Q_{f} \text { and } \exists \lambda>0: \mathscr{F}\left(\sigma^{1}(x)\right) \leq-\lambda \text { for a.a. } x \in \Omega
$$

holds, then the Problem ( $\mathscr{P}^{*}$ ) has a unique solution, and the following equality is valid (see, for instance, [3]):

$$
\inf (\mathscr{P})=R(\sigma)=\sup \left(\mathscr{P}^{*}\right) .
$$

Unfortunately, the Problem $(\mathscr{P})$ in general is not solvable and, therefore, it must be relaxed. The corresponding variational relaxation was made in [1, 7 and 12]. Its weak solution belongs to the space $B D(\Omega)$ of vector-valued functions of bounded deformation (see, for instance, [13]). way.

The problem which is dual to Problem $\left(\mathscr{P}_{\delta}\right)$ may be posed in the following

Problem $\left(\mathscr{P}_{\delta}^{*}\right)$ : Find $\sigma^{\delta} \in Q_{f}$ such that

$$
R_{\delta}\left(\sigma^{\delta}\right)=\sup \left\{R^{\delta}(\tau): \tau \in Q_{f}\right\}
$$

where

$$
R_{\delta}(\tau):=R(\tau)-\frac{1}{2}\left(\frac{1}{\delta}-\frac{1}{2 \mu}\right) \int_{\Omega}\left(\left|\sigma^{D}\right|-\sqrt{2} k_{*}\right)_{+}^{2} d x
$$

and $(z)_{+}$is the nonnegative part of $z$.

Problem $\left(\mathscr{P}_{\delta}^{*}\right)$ is uniquely solvable as well, and

$$
J_{\delta}\left(u^{\delta}\right)=R_{\delta}\left(\sigma_{\delta}\right)
$$

Concerning the relation between Problem $\left(\mathscr{P}_{\delta}\right)$ and Problem $\left(\mathscr{P}_{\delta}^{*}\right)$ we have the following result: 
Theorem 1. Suppose that the conditions (2.2), (2.5) hold. Then

$$
\begin{gathered}
R_{\delta}\left(\sigma^{\delta}\right) \rightarrow R(\sigma) \text { as } \delta \rightarrow 0, \\
\frac{1}{2} \int_{\Omega} a\left(\sigma^{\delta}-\sigma, \sigma^{\delta}-\sigma\right) d x \leq R_{\delta}\left(\sigma^{\delta}\right)-R(\sigma) .
\end{gathered}
$$

Proof. In view of the inequalities

we have

$$
g_{0}(t) \leq g_{0 \delta}(t) \leq g_{0 \mu}(t) \quad \text { for all } t \in \mathbb{R} \text { and } \delta \in(0, \mu]
$$

$$
\inf _{v \in V_{0}+u_{0}} I(v) \leq \inf _{v \in V_{0}+u_{0}} I_{\delta}(v)=I_{\delta}\left(u^{\delta}\right) \leq I_{\delta}\left(u_{0}\right) \leq I_{\mu}\left(u_{0}\right)
$$

Thus, the equality (1.6) gives us the following estimates:

$$
\delta \int_{\Omega}\left|\varepsilon^{D}\left(u^{\delta}\right)\right|^{2} d x \leq C_{1}, \quad \int_{\Omega} \operatorname{div}^{2} u^{\delta} d x \leq C_{2}, \quad \int_{\Omega}\left|\varepsilon\left(u^{\delta}\right)\right| d x<C_{3}
$$

with positive constants $C_{i}$ which do not depend on $\delta$.

Next, it is clear that the necessary and sufficient condition for the minimum of the problem may be written in the form

$$
\int_{\Omega} \sigma^{\delta}: \varepsilon(v) d x=M(v) \quad \text { for all } v \in V^{0}
$$

where

$$
\begin{aligned}
\sigma^{\delta} & =\frac{\partial g}{\partial \varepsilon}\left(\varepsilon\left(u^{\delta}\right)\right)=\delta \varepsilon^{D}\left(u^{\delta}\right) \mathbb{I}+K_{0} \operatorname{div} u^{\delta} \mathbb{I}+\frac{2 \mu-\delta}{2 \mu} g_{0 \delta}^{\prime}\left(\left|\varepsilon^{D}\left(u^{\delta}\right)\right|\right) \frac{\varepsilon^{D}\left(u^{\delta}\right)}{\left|\varepsilon^{D}\left(u^{\delta}\right)\right|} \\
& =\delta \varepsilon^{D}\left(u^{\delta}\right)+\frac{\delta}{2 \mu} K_{0} \operatorname{div} u^{\delta} \mathbb{I}+\frac{2 \mu-\delta}{2 \mu} \frac{\partial g}{\partial \varepsilon}\left(\varepsilon\left(u^{\delta}\right)\right) .
\end{aligned}
$$

Setting

we can assert that

$$
\sigma_{0}^{\delta}:=\frac{2 \mu}{2 \mu-\delta}\left(\sigma^{\delta}-\delta \varepsilon^{D}\left(u^{\delta}\right)-\frac{\delta}{2 \mu} K_{0} \operatorname{div} u^{\delta} \mathbb{I}\right)
$$

$$
\sigma_{0}^{\delta} \in K
$$

The theory of duality gives the extremality relation

$$
\sigma_{0}^{\delta}: \varepsilon\left(u^{\delta}\right)-g\left(\varepsilon\left(u^{\delta}\right)\right)-\frac{1}{2} a\left(\sigma^{\delta}, \sigma^{\delta}\right)=0 \text { a.e. in } \Omega \text {. }
$$

Taking into account the equilibrium equations (2.12) and the estimates (2.11), we arrive at

$$
\begin{aligned}
I_{\delta}\left(u^{\delta}\right)= & \int_{\Omega}\left\{\frac{\delta}{2}\left|\varepsilon^{D}\left(u^{\delta}\right)\right|^{2}+\sigma_{0}^{\delta}: \varepsilon\left(u^{\delta}\right)-\frac{1}{2} a\left(\sigma_{0}^{\delta}, \sigma_{0}^{\delta}\right)\right\} d x-M\left(u^{\delta}\right) \\
= & \int_{\Omega} \sigma_{0}^{\delta}: \varepsilon\left(u^{\delta}-u_{0}\right) d x-M\left(u^{\delta}-u_{0}\right)+\frac{\delta}{2} \int_{\Omega}\left|\varepsilon^{D}\left(u^{\delta}\right)\right|^{2} d x+R\left(\sigma_{0}^{\delta}\right) \\
= & \frac{2 \mu}{2 \mu-\delta} \int_{\Omega}\left(\sigma^{\delta}-\delta \varepsilon^{D}\left(u^{\delta}\right)-\frac{\delta}{2 \mu} K_{0} \operatorname{div} u^{\delta} \mathbb{I}\right): \varepsilon\left(u^{\delta}-u_{0}\right) d x \\
& -M\left(u^{\delta}-u_{0}\right)+\frac{\delta}{2} \int_{\Omega}\left|\varepsilon^{D}\left(u^{\delta}\right)\right|^{2} d x+R\left(\sigma_{0}^{\delta}\right) \\
= & \beta(\delta)+R\left(\sigma_{0}^{\delta}\right)-\frac{\delta}{2} \int_{\Omega}\left|\varepsilon^{D}\left(u^{\delta}\right)\right|^{2} d x \\
\leq & \beta(\delta)+R\left(\sigma_{0}^{\delta}\right),
\end{aligned}
$$


where $\beta(\delta) \rightarrow 0$ as $\delta \rightarrow 0$. Presupposing that the statement (2.9) is false, we have

$$
A:=\lim _{\delta \rightarrow 0} \sup R_{\delta}\left(\sigma^{\delta}\right)>R(\sigma)
$$

Then there are sequences such that

$$
\begin{aligned}
& R_{\delta_{k}}\left(\sigma^{\delta_{k}}\right) \rightarrow A, \\
& \sigma_{0}^{\delta_{k}} \rightarrow \sigma_{0} \in K \quad \text { weakly in } L^{2}\left(\Omega ; M_{s}^{n \times n}\right) .
\end{aligned}
$$

It is very easy to see that $\sigma_{0} \in Q_{f}$. By the upper semicontinuity of the functional $R$ on $L^{2}\left(\Omega ; M_{s}^{n \times n}\right)$ we get from $(2.15),(2.16)$ and (2.17)

$$
R(\sigma)<A \leq R\left(\sigma_{0}\right), \sigma_{0} \in Q_{f} \cap K \Longrightarrow R\left(\sigma_{0}\right) \leq R(\sigma) .
$$

So, we obtain a contradiction. We now prove the statement (2.10). A necessary and sufficient condition for the extremum of the Problem $\left(\mathscr{P}_{\delta}^{*}\right)$ has the form

$$
\begin{aligned}
\int_{\Omega}\left\{a\left(\sigma^{\delta}, \tau-\sigma^{\delta}\right)+\left(\frac{1}{\delta}-\frac{1}{2 \mu}\right)\left(\left|\sigma^{\delta D}\right|\right.\right. & \left.-\sqrt{2} k_{*}\right)_{+} \frac{\sigma^{\delta D}:\left(\tau^{D}-\sigma^{\delta D}\right)}{\left|\sigma^{\delta D}\right|} \\
& \left.-\varepsilon\left(u_{0}\right):\left(\tau-\sigma^{\delta}\right)\right\} d x=0
\end{aligned}
$$

for all $\tau \in Q_{f}$. Thus, we can write

$$
\begin{aligned}
R(\sigma)- & R_{\delta}\left(\sigma^{\delta}\right)=\int_{\Omega}\left\{-\frac{1}{2} a(\sigma, \sigma)+\varepsilon\left(u_{0}\right): \sigma+\frac{1}{2} a\left(\sigma^{\delta}, \sigma^{\delta}\right)-\varepsilon\left(u_{0}\right): \sigma^{\delta}\right. \\
& \left.+\frac{1}{2}\left(\frac{1}{\delta}-\frac{1}{2 \mu}\right)\left(\left|\sigma^{\delta D}\right|-\sqrt{2} k_{*}\right)_{+}^{2}\right\} d x \\
= & \int_{\Omega}\left\{-\frac{1}{2} a\left(\sigma^{\delta}-\sigma, \sigma^{\delta}-\sigma\right)-a\left(\sigma^{\delta}, \sigma-\sigma^{\delta}\right)+\varepsilon\left(u_{0}\right):\left(\sigma-\sigma^{\delta}\right)\right. \\
= & \left.\left.-\frac{1}{2} \int_{\Omega} a\left(\sigma-\sigma^{\delta}, \sigma-\sigma^{\delta}\right) d x-\frac{1}{2 \mu}\right)\left(\left|\sigma^{\delta D}\right|-\sqrt{2} k_{*}\right)_{+}^{2}\right\} d x \\
& +\left(\frac{1}{\delta}-\frac{1}{2 \mu}\right) \int_{\Omega}\left(\left|\sigma^{\delta D}\right|-\sqrt{2} k_{*}\right)_{+} \frac{\sigma^{\delta D}:\left(\sigma^{D}-\sigma^{\delta D}\right)}{\left|\sigma^{\delta D}\right|} d x \\
& +\frac{1}{2}\left(\frac{1}{\delta}-\frac{1}{2 \mu}\right) \int_{\Omega}\left(\left|\sigma^{\delta D}\right|-\sqrt{2} k_{*}\right)_{+}^{2} d x \\
\leq & -\int_{\Omega} \frac{1}{2} a\left(\sigma-\sigma^{\delta}, \sigma-\sigma^{\delta}\right) d x+\frac{1}{2}\left(\frac{1}{\delta}-\frac{1}{2 \mu}\right) \int_{\Omega}\left(\left|\sigma^{\delta D}\right|-\sqrt{2} k_{*}\right)_{+}^{2} d x \\
= & -\frac{1}{2} \int_{\Omega} a\left(\sigma-\sigma^{\delta}, \sigma^{\delta}-\sigma\right) d x .
\end{aligned}
$$

The assertion (2.10) is proved.

\section{DUAL FINITE ELEMENT APPROXIMATION AND ERROR ESTIMATE}

To avoid some technical difficulties, we consider the case when $f=0, \partial_{2} \Omega=$ 0 and $\Omega$ is a polygon or a polyhedron. Then $Q:=Q_{f}$ is transformed into the subspace

$$
Q:=\left\{r \in L^{2}\left(\Omega ; M_{s}^{n \times n}\right): \operatorname{div} \tau=0\right\} .
$$


Let $Q^{h}$ be some finite-dimensional subspace in $\Omega$. We consider the following finite-dimensional problem.

Problem $\left(\mathscr{P}_{\delta}^{* h}\right)$ : Find $\sigma^{\delta, h} \in Q^{h}$ such that

$$
R_{\delta}\left(\sigma^{\delta, h}\right)=\sup \left\{R_{\delta}\left(\tau^{h}\right): \tau^{h} \in Q^{h}\right\} .
$$

Theorem 2. The following error estimate is valid:

$$
\left\|\sigma^{\delta, h}-\sigma\right\|_{L^{2}\left(\Omega ; M_{s}^{n \times n}\right)}^{2} \leq C\left(\frac{1}{\sqrt{\delta}} \inf _{\tau^{h} \in Q^{h}}\left\|\tau^{h}-\sigma^{\delta}\right\|_{L^{2}\left(\Omega ; M_{s}^{n \times n)}\right.}+R_{\delta}\left(\sigma^{\delta}\right)-R(\sigma)\right) .
$$

Here, the positive constant $C$ does not depend on $h, \delta$ and $\sigma$.

Proof. A necessary and sufficient condition for the maximum of the Problem $\left(\mathscr{P}_{\delta}^{* h}\right)$ looks formally like $(2.19)$ :

$$
\begin{aligned}
\int_{\Omega}\left\{a \left(\sigma^{\delta, h},\right.\right. & \left.\tau^{h}-\sigma^{\delta, h}\right)-\varepsilon\left(u_{0}\right):\left(\tau^{h}-\sigma^{\delta, h}\right) \\
& \left.+\left(\frac{1}{\delta}-\frac{1}{2 \mu}\right)\left(\left|\sigma^{\delta, h D}\right|-\sqrt{2} k_{*}\right)_{+} \frac{\sigma^{\delta, h D}:\left(\tau^{h D}-\sigma^{\delta, h D}\right)}{\left|\sigma^{\delta D}\right|}\right\} d x=0
\end{aligned}
$$

for all $\tau^{h} \in Q^{h}$. Setting $\tau^{h}=0$ in (3.3), we obtain the energy estimate

$$
\int_{\Omega}\left\langle a\left(\sigma^{\delta, h}, \sigma^{\delta, h}\right)+\left(\frac{1}{\delta}-\frac{1}{2 \mu}\right)\left(\left|\sigma^{\delta, h D}\right|-\sqrt{2} k_{*}\right)_{+}^{2}\right\rangle d x \leq C_{4},
$$

where the positive constant $C_{4}$ depends only on $\left\|\varepsilon\left(u_{0}\right)\right\|_{L^{2}\left(\Omega ; M_{s}^{n \times n}\right)}$. By the equations (2.19) and (3.3), we have

$$
\begin{aligned}
& \int_{\Omega}\left\{a\left(\sigma^{\delta, h}-\sigma^{\delta}, \sigma^{\delta, h}-\sigma^{\delta}\right)\right. \\
& +\left(\frac{1}{\delta}-\frac{1}{2 \mu}\right)\left(\left(\left|\sigma^{\delta D}\right|-\sqrt{2} k_{*}\right)_{+} \frac{\sigma^{\delta D}}{\left|\sigma^{\delta D}\right|}\right. \\
& \left.\left.\quad-\left(\left|\sigma^{\delta, h D}\right|-\sqrt{2} k_{*}\right)_{+} \frac{\sigma^{\delta, h D}}{\left|\sigma^{\delta, h D}\right|}\right):\left(\sigma^{h D}-\sigma^{\delta, h D}\right)\right\} d x \\
& =\int_{\Omega}\left\{a\left(\sigma^{\delta, h}, \tau^{h}-\sigma^{\delta}\right)-\varepsilon\left(u_{0}\right):\left(\tau^{h}-\sigma^{\delta}\right)\right. \\
& \left.\quad+\left(\frac{1}{\delta}-\frac{1}{2 \mu}\right)\left(\left|\sigma^{\delta, D}\right|-\sqrt{2} k_{*}\right)_{+} \frac{\sigma^{\delta, h D}:\left(\tau^{h}-\sigma^{\delta}\right)}{\left|\sigma^{\delta, h D}\right|}\right\} d x .
\end{aligned}
$$

It follows from the previous equality that

$$
\begin{aligned}
\int_{\Omega} a\left(\sigma^{\delta, h}-\sigma^{\delta}, \sigma^{\delta, h}-\sigma^{\delta}\right) d x \\
\leq C_{5}\left(\left\|\sigma^{\delta, h}\right\|_{L^{2}\left(\Omega ; M_{s}^{n \times n}\right)}+\left\|\varepsilon\left(u_{0}\right)\right\|_{L^{2}\left(\Omega ; M_{s}^{n \times n}\right)}\right. \\
\left.\quad+\left(\frac{1}{\delta}-\frac{1}{2 \mu}\right)\left\|\left(\left|\sigma^{\delta, D}\right|-\sqrt{2} k_{*}\right)_{+}\right\|_{L^{2}(\Omega)}\right)\left\|\tau^{h}-\sigma^{\delta}\right\|_{L^{2}\left(\Omega ; M_{s}^{n \times n}\right)} .
\end{aligned}
$$

Thus, the required estimate (3.2) follows from (2.10) and (3.4).

Remark. For $n=2$ one can find in [6] some useful examples of the subspace $Q^{h}$. Corresponding finite element approximations are affine at each element. 
The simplest way of constructing such elements is based on introducing Airy's stress function and applying the composite piecewise cubic plate bending elements of Hsieh-Clough-Tocher (see, for instance, [2]).

Suppose that

$$
\left\|\left|\nabla \sigma^{\delta}\right|\right\|_{L^{2}} \leq C_{1}
$$

with a constant $C_{1}$ which does not depend on $\delta$. Then for the finite element approximation in question we can state

$$
\inf _{\tau^{h} \in Q^{h}}\left\|\tau^{h}-\sigma^{\delta}\right\|_{L^{2}\left(\Omega ; M_{s}^{n \times n}\right)} \leq C_{2} h \quad(\text { does not depend on } \delta) .
$$

We choose $\delta=h$ in (3.6) and (3.2). For this case the following estimate holds:

$$
\left\|\sigma^{h, h}-\sigma\right\|_{L^{2}\left(\Omega ; M_{s}^{n \times n}\right)} \leq C_{3} \sqrt{h}+R_{h}\left(\sigma^{h}\right)-R(\sigma) .
$$

Sometimes it is possible to find estimates for $R_{h}\left(\sigma^{h}\right)-R(\sigma)$ from the mechanical point of view or as a posteriori estimates.

Unfortunately, the authors have failed to prove the estimate (3.5), but its local variant has been proved in [10] and [11], i.e.,

$$
\left\|\left|\nabla \sigma^{\delta}\right|\right\|_{L^{2}\left(\Omega_{0}\right)} \leq C_{4} \quad \forall \Omega_{0} \Subset \Omega
$$

with a constant $C_{4}$ which depends on $\operatorname{dist}\left(\Omega_{0}, \partial \Omega\right.$ ) but not on $\delta$.

The proposed dual finite element method gives directly approximations for stresses and corresponding plastic and elastic zones. In the primal finite element method it is necessary to calculate first derivatives of displacements to get the corresponding information.

For other approaches of finite element approximations to the solution of the Problem $\left(\mathscr{P}^{*}\right)$ we refer, for instance, to $[4,8]$ and, in optimal shape design context, to [5].

\section{BIBLIOGRAPHY}

1. G. Anzellotti and M. Giaquinta, On the existence of the fields of stresses and displacements for an elasto-perfectly plastic body in static equilibrium, J. Math. Pures Appl. 61 (1982), 219-244.

2. P. Ciarlet, The finite element method for elliptic problems, North-Holland, Amsterdam, 1978.

3. G. Duvaut and J. L. Lions, Les inéquations en mécanique et en physique, Dunod, Paris, 1972.

4. I. Hlaváček, J. Haslinger, J. Nečas, and J. Lovišek, Numerical solution of variational inequalities in mechanics, Springer-Verlag, New York, 1988.

5. J. Haslinger and P. Neittaanmäki, Finite element approximation of optimal shape design: Theory and applications, Wiley, Chichester, 1988.

6. C. Johnson and B. Mercier, Some equilibrium finite element methods for two-dimensional elasticity problems, Numer. Math. 30 (1978), 103-116.

7. R. Kohn and R. Temam, Dual spaces of stresses and strains with application to Hencky plasticity, Appl. Math. Optim. 10 (1983), 1-35.

8. V. Rivkind, L. Rukhovetz, and L. Oganesjan, Variational-difference schemes, J. Lit. Acad. Sci. Vilnius 1 (1971), 2 (1973).

9. G. Seregin, On the well-posedness of variational problems of mechanics of ideally elastic-plastic media, Soviet Phys. Dokl. 5 (1984), 316-318. 
10. _ Differentiability of local extremals of variational problems in the mechanics of perfect elastoplastic media, Differential Equations 23 (1987), 1981-1991.

11. _ On the regularity of weak solutions of variational problems in plasticity theory, Leningrad Math. J. 2 (1991), 321-337.

12. __ Variation-difference scheme for problems on the mechanics of ideally elasto-plastic media, USSR Comput. Math. and Math. Phys. 25 (1985), 153-165.

13. R. Temam and G. Strang, Functions of bounded deformation, Arch. Rational Mech. Anal. 75 (1980), 7-21.

(Neittaanmäki) Department OF MAThematics, UNiVersity OF JYVÄSKYLÄ, SF-40100 JYVÄSKYLÄ, FinLAND

(Rivkind and Seregin) Department of Mathematics, St. Petersburg University, Petrodvoretz Bibliotechnaja Square 2, St. Petersburg 199034, Russia 